\title{
LA ELECCIÓN PARLAMENTARIA DEL PRESIDENTE DEL GOBIERNO EN ESPAÑA: ANÁLISIS NORMATIVO, ESTABILIDAD INSTITUCIONAL Y PROPUESTA DE REFORMA DEL ARTÍCULO 99.5 DE LA CONSTITUCIÓN ESPAÑOLA
}

The parliamentary election of the president of the Government in Spain: Regulatory analysis, institutional stability, and proposal for reform of article 99.5 of the Spanish Constitution

\author{
ÓSCAR MATEOS Y DE CABO \\ Universidad Rey Juan Carlos \\ oscar.mateos@urjc.es
}

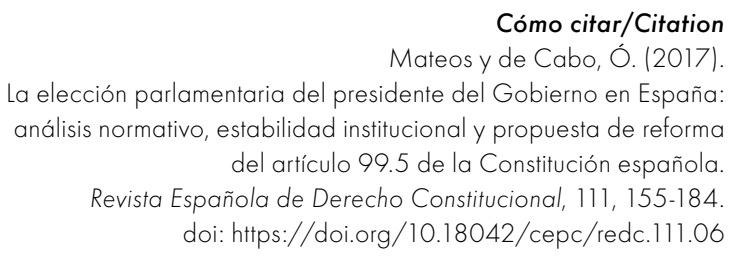

Resumen

La configuración constitucional del art. 99 de la Constitución española de 1978 permite un sistema de elección parlamentaria, del presidente del Gobierno, sencillo cuando los resultados electorales garantizan la mayoría absoluta o simple en el Congreso de los Diputados. Sin embargo, el fracaso de la XI Legislatura en la formación de un Gobierno ha puesto de relieve problemas sin precedente, y no detectados anteriormente, en el actual período democrático en España. En primer lugar, la falta de acuerdo parlamentario para la formación de Gobierno, con la necesidad de convocar nuevas elecciones. En segundo lugar, un posible bloqueo institucional al que se puede llegar si ningún candidato a presidente del Gobierno se presenta a la investidura en el Congreso de los Diputados.

En este trabajo de investigación se analiza y reflexiona sobre estas y otras cuestiones, de gran gravedad y transcendencia política e institucional. La formación de 
Gobierno es una de las principales funciones parlamentarias, y sus repercusiones negativas, por la falta de acuerdo, son evidentes. Por otro lado, la posibilidad de que ningún candidato se presente a la investidura bloquearía las previsiones del art. 99.5 de la Constitución.

Por tanto, en este trabajo de investigación se estudia la doctrina, la jurisprudencia y la normativa relevante. Se propone la reforma constitucional del art. 99.5 de la Constitución y se realizan una serie de propuestas para la estabilidad institucional y política, que se formulan a lo largo del estudio, sintetizadas en el último apartado de conclusiones.

\section{Palabras clave}

Presidente del Gobierno; sesión de investidura; formación del Gobierno; confianza del Congreso de los Diputados; responsabilidad parlamentaria; estabilidad institucional; reforma del art. 99.5 de la Constitución.

\section{Abstract}

The configuration constitutional art. 99 of the Spanish Constitution of 1978 allows a simple system of parliamentary election of President of Government, when the election results guarantee absolute or simple majority in the Congress of Deputies. However, the failure of the XI Legislature in forming a government has highlighted problems unprecedented, not previously detected, in the current democratic period in Spain. First, the lack of parliamentary agreement to form government with the need to call new elections. Second, a possible institutional blockage, which can be reached if no candidate for President of Government is presented to the investiture in the Congress of Deputies.

In this research, we analyse and reflect on these and other issues of great seriousness and of political and institutional significance. Government formation is one of the main parliamentary functions and its negative impact, for the lack of agreement, is evident. On the other hand, the possibility that no candidate is presented to the investiture can block the provisions of art. 99.5 of the Constitution.

Therefore, in this research the doctrine, jurisprudence and relevant legislation is studied. The constitutional reform of art. 99.5 of the Constitution is proposed, and a number of proposals for institutional and political stability, which are made throughout the study, and they are summarized in the final section of conclusions.

\section{Keywords}

President of Government; investiture session; formation of the Government; confidence of the Congress of Deputies; parliamentary accountability; institutional stability; reform of art. 99.5 of the Constitution. 
I. PLANTEAMIENTO GENERAL. II. EL NOMBRAMIENTO ORDINARIO DEL PRESIDENTE DEL GOBIERNO. III. EL PAPEL DEL REY EN LA ELECCIÓN DEL PRESIDENTE DEL GOBIERNO. IV. LA SESIÓN DE INVESTIDURA: PERFILES CONSTITUCIONALES Y REGLAMENTARIOS. V. LAS DIFERENTES FORMAS DE INVESTIDURA EN LOS ESTATUTOS DE AUTONOMÍA. VI. LA PROPUESTA DE REFORMA CONSTITUCIONAL DEL ART. 99.5 CE. VII. CONCLUSIONES. BIBLIOGRAFÍA.

\section{PLANTEAMIENTO GENERAL}

El procedimiento de investidura es, sin duda, una parte muy importante del proceso de la formación del Gobierno en muchos países, sin embargo, Robert Zbíral (2011: 58) señala que los trabajos científicos que se dedican al tema son todavía demasiado escasos en relación a su enorme transcendencia sobre el buen funcionamiento del sistema político e institucional.

La confianza expresa o tácita del primer ministro es consecuencia de un proceso histórico, en el que esta figura deja de ser un simple secretario del rey para ir ganando protagonismo, primero como un primus inter pares $\mathrm{y}$, posteriormente, constituirse en la persona que configura y dirige el Gobierno, un órgano que gana en relevancia y funciones determinando, precisamente, en su relación con el poder legislativo, uno de los rasgos más peculiares del sistema parlamentario.

Sin embargo, en algunos Estados con régimen parlamentario, como Gran Bretańa, Holanda o Dinamarca, el Parlamento no se pronuncia a priori mediante una votación de investidura para otorgar su confianza al primer ministro, pues se acepta la presunción de que el Ejecutivo, salido de la mayoría parlamentaria de las últimas elecciones, cuenta con la confianza parlamentaria, mientras que las Cámaras no insten una moción de censura u otra propuesta que implique la falta de confianza en relación al Gabinete.

No obstante, a pesar de esta premisa, en la que se basa el llamado parlamentarismo negativo, la costumbre en algunos países, como en Bélgica y, también, las previsiones constitucionales en Italia, requieren, sin embargo, al nuevo Gobierno a someterse a las votaciones en las Cámaras para lograr la investidura, que en este último caso sería dentro de los diez días siguientes a su formación (art. 94 de la Constitución de la República italiana de 1947). 
De esta manera, la formación del Gobierno en los citados casos se constituye como un procedimiento previo y autónomo, que no precisa del voto de confianza afirmativo de las Cámaras para entrar en funciones, lo que no evita que este se pueda exigir en otros países, como en Italia, a posteriori, ni la polémica doctrinal sobre el alcance de sus competencias, con anterioridad a su investidura parlamentaria, poniendo de relieve las variadas y complejas relaciones institucionales entre el Gobierno y el Parlamento, en un régimen que se ha caracterizado como de colaboración pero en el que se ha puesto de manifiesto la tendencia hacia el reforzamiento del papel del Ejecutivo (Pisaneschi, 2015: 1) e, incluso, se ha llegado a hablar de una cierta pugna sobre el protagonismo de estas instituciones (Perini, 2009).

Por otro lado, en otros sistemas constitucionales se sigue el llamado patrón del parlamentarismo positivo, como en el caso del voto de investidura recogido en el art. 68 de la Constitución de la República Checa de 1992, que consagra un modelo de responsabilidad gubernamental ante la Cámara de Diputados (Zbíral, 2011: 73).

El parlamentarismo positivo también caracteriza el sistema de investidura en Hungría entendiendo por una de sus notas distintivas la manifestación de la confianza parlamentaria, que requiere una aprobación explícita del Parlamento para que el Gobierno pueda formarse y asumir sus funciones (Horváth, 2011:7).

No faltan países que han pasado de un sistema de confianza tácito, hacia el Gobierno, a otro modelo de constatación expresa, emitida por el Parlamento. Este es el caso de Irlanda, que históricamente se encontraba bajo la influencia británica pero que ha derivado a un régimen de "confianza expresa» cuando se ha exigido una votación de investidura, tal y como se recogió en la Constitución Provisional de 1919 (Martin, 2011:3).

De esta forma, con la investidura se pone de relieve el deseo del Parlamento «rebelde» irlandés de asegurar que el monarca británico no tendría ningún papel en la determinación o el nombramiento del jefe de Gobierno. En consecuencia, esta decisión la tomarían los miembros de la Cámara Baja de Irlanda (Dáil), que serían los únicos con la capacidad de nominar y destituir al jefe de Gobierno, y de aprobar las leyes. Posteriormente, el art. 53 de la Constitución de 1922 ha sido taxativo al establecer que «el Presidente del Consejo será nombrado por la designación del Dáil Éireann».

En la posterior Constitución irlandesa de 1937 los constituyentes podrían haber adoptado un sistema similar al del jefe del Estado de Gran Bretańa, a la hora de proceder a la elección del primer ministro. Sin embargo, aparte de nombrar formalmente a las personas designadas por el Dáil, el presidente de la República no juega ningún papel en la formación del Gobierno, ni seleccionando a 
las personas para ocupar cargos ni participando en las funciones ejecutivas. De esta forma, el art. 13.1.2 de la Constitución de 1937 establece que el presidente de la República nombre a los demás miembros del Gobierno, pero con la previa designación del presidente del Gobierno y con la aprobación previa de Dáil.

Por otro lado, desde el punto de vista del derecho constitucional comparado, el nombramiento del Gobierno, en el sistema parlamentario, puede encuadrarse básicamente dentro de tres grandes formulaciones:

1. En el nombramiento por el jefe del Estado, sin intervención del Parlamento, que es propio de los países de parlamentarismo negativo, tal y como sucede en las monarquías del norte europeo, y cuya función es garantizar la estabilidad de Gobiernos en minoría. Es decir, se presenta la implantación de dicho modelo como un instrumento de racionalización parlamentaria, que permite que un Gobierno se forme y perdure mientras el Parlamento no le retire de manera expresa su apoyo.

2. En el nombramiento del presidente del Gobierno por el jefe del Estado y posterior intervención del Parlamento, como sucede en Bélgica o Italia.

3. Por último, en el nombramiento del presidente del Ejecutivo por el Parlamento, sin intervención del jefe del Estado, como sucede en Japón o en Irlanda.

En el modelo español la confianza la otorga el Congreso de los Diputados, en la sesión de investidura, y el rey procede posteriormente al nombramiento como presidente del Gobierno, si bien este acto regio es formal y debido pues el protagonismo a la hora de otorgar la confianza la tiene el Congreso de los Diputados, lo que es claramente visible en la configuración del propio art. 99.2 CE.

Esta regulación constitucional no constituye únicamente un mero procedimiento formal, sino que tiene detrás un amplio y largo bagaje, propio del funcionamiento de los regímenes parlamentarios que, entre otras implicaciones, dota de cierta estabilidad institucional a la función ejecutiva, al contar con el respaldo parlamentario. En este sentido, consideramos, al igual que lo hace Sánchez Navarro (1995: 291), que la crisis del parlamentarismo deriva, en gran parte, de una inadecuada comprensión de cuál debe ser precisamente la articulación de las instituciones en los regímenes parlamentarios. El enfoque debe ser complejo, pues sobre esta realidad no solo inciden los esquemas formales que, analizados aisladamente, no consiguen explicar satisfactoriamente el desarrollo del sistema político, debido a que no en todos los casos la configuración formal 
preside y sirve de cauce a la práctica política y parlamentaria (Sánchez Navarro, 1997: 349).

En este contexto, estudiaremos los efectos que sobre nuestro sistema constitucional y político tuvieron los resultados de las elecciones generales del 20 de diciembre de 2015, con las que se inicia la XI legislatura ${ }^{1}$, caracterizada por una situación insólita que ha permitido la constitución de las dos Cámaras parlamentarias pero que no ha conseguido que el candidato propuesto por el rey alcanzase la mayoría suficiente para formar Gobierno.

En las segundas elecciones generales, del 26 de junio de 2016, finalmente se llega a un pacto, que permite a Mariano Rajoy ser investido, en una segunda votación, presidente del Gobierno, el 29 de octubre de 2016, gracias al voto favorable de su partido, Ciudadanos, Coalición Canaria, Foro Asturias y Unión del Pueblo Navarro.

En estos acontecimientos han influido, decisivamente, las reglas de la investidura parlamentaria en España, pues los requisitos formales requeridos para la elección del presidente del Gobierno y, posteriormente, la de su equipo ministerial funcionan casi de forma mecánica en la aritmética de las votaciones parlamentarias, en condiciones de mayorías sólidas, pero implican otras consecuencias institucionales y políticas en el caso de no poder superar alguno de los procedimientos de investidura, que se suelen requerir en el ámbito del derecho comparado, es decir, la mayoría absoluta o la mayoría relativa de la Cámara (De Winter: 1995).

Estos problemas pueden suceder también en otros países como, por ejemplo, en las últimas elecciones generales de Irlanda, de 26 de febrero de 2016, que originaron un bloqueo político que se denominó en la prensa, como «a la española», debido a que la coalición gubernamental saliente no lograba alcanzar la mayoría y la oposición rechazaba pactar. En un titular de prensa se ha recogido la noticia con el título: «Los comicios llevan a Irlanda a un bloqueo político a la española». Sin embargo, el 29 de abril, finalmente, los dos principales partidos llegaron a un acuerdo para constituir un Gobierno en minoría ${ }^{2}$.

Para desbloquear este tipo de problemas institucionales y políticos, la investidura o las reglas que se fijen para la concesión de la confianza parlamentaria al Gobierno serán determinantes para la formación del futuro

1 La XI Legislatura del Parlamento español se inicia el 13-12-2016 y finaliza el 3-52016 con una duración de 111 días, la más corta del periodo democrático.

2 Véase: «Fine Gael and Fianna Fáil finally reach minority government deal». Independent.ie. 29-4-2016. Véase: http://www.independent.ie/irish-news/election-2016/news/ fine-gael-and-fianna-fil-finally-reach-minority-government-deal-34672359.html. 
Gobierno. Se trata claramente de un proceso que, formalmente, es secuencial, pues el requisito previo de la confianza parlamentaria del presidente del Gobierno constituye un requisito sine qua non para la subsiguiente formación del órgano colegiado.

En este sentido, además de analizar el régimen jurídico del procedimiento de elección parlamentaria, del candidato a la presidencia del Gobierno, serán pertinentes algunas observaciones sobre la labor de liderazgo político e institucional que debe desempeñar la persona que se postula, puesto que el papel institucional y político desempeñado por el candidato a la presidencia del Gobierno será fundamental para conseguir los consensos necesarios que permitan el buen funcionamiento de los mecanismos constitucionales, de lo que no solo va a depender la futura dirección gubernamental, sino también del propio conjunto del complejo estatal (Mateos de Cabo, 2006: 416).

En este sentido, resaltamos la caracterización tan acertada de Aragón Reyes, cuando afirma que en España el sistema parlamentario no es de «Gabinete», sino de "Canciller» o de "Primer Ministro». Por tanto, nuestra Constitución ha optado claramente "por un modelo de parlamentarismo racionalizado mediante el establecimiento de determinadas reglas que, de un lado, favorecen la estabilidad gubernamental y, de otro, realzan notoriamente la figura del Presidente del Gobierno» (Aragón Reyes, 2005).

Por eso, nuestra Constitución de 1978 exige la previa investidura del presidente por el Congreso de los Diputados como necesaria para la formación del Gobierno, de modo que solo tras la obtención de la confianza parlamentaria del presidente pueden dictarse los decretos de nombramiento del equipo gubernamental, al constatarse expresamente que cuenta con la confianza de la Cámara para gobernar.

Es decir, el modelo es indiscutiblemente de formación «parlamentaria explícita» del Gobierno, en la línea de lo establecido por la Constitución francesa de 1946, por la Ley Fundamental de Bonn de 1949, por la Constitución japonesa de 1946 o por la sueca de 1974 . En consecuencia, en nuestro régimen constitucional la investidura del presidente del Gobierno es una fase imprescindible y necesaria para la formación del Gobierno, que se justifica fundamentalmente por las razones a las que antes hemos hecho referencia.

\section{EL NOMBRAMIENTO ORDINARIO DEL PRESIDENTE DEL GOBIERNO}

La Constitución de 1978 establece para el nombramiento del presidente del Gobierno dos procedimientos bien diferenciados de designación. Según la terminología de Sánchez Agesta nos estamos refiriendo al nombramiento 
«ordinario» del nuevo Gobierno, cuando este se produce siguiendo la detallada regulación contenida en el art. $99 \mathrm{CE}$.

Por otro lado, nos encontramos ante el procedimiento de nombramiento extraordinario, en el caso de una moción de censura que tiene, como particularidad sobre el procedimiento anterior, que ajustarse a un trámite abreviado y excepcional, pero que presupone que se han cumplido, previamente, los requisitos exigidos en el art. $99 \mathrm{CE}$ para el nombramiento ordinario (Sánchez Agesta, 1991: 274-275).

La llamada moción de censura constructiva, que encuentra su origen en el constitucionalismo alemán, no representa un bloqueo institucional para la investidura de un nuevo presidente del Gobierno, pues para que prospere la moción es necesario que el Parlamento exprese, por propia iniciativa, la ruptura de la relación de confianza que le vinculaba con el anterior Gobierno, que, en el caso español, supone su cese y la designación de un nuevo Gobierno, con investidura automática del nuevo presidente del mismo (Montero Giber, 1984: 555).

De esta forma, la moción de censura, que nuestra Constitución regula en los arts. 113 y $114 \mathrm{CE}$, pertenece al tipo denominado de "censura constructiva», y dentro de este se caracteriza por ser especialmente favorable a la conservación del Gobierno, lo cual no siempre es lo mismo que la estabilidad política, ya que puede estar «muy condicionada por el sistema de partidos y por la disciplina parlamentaria en las Cámaras» ${ }^{3}$.

Por estas razones no nos vamos a ocupar en nuestro estudio sobre la moción de censura pues, aunque es cierto que implica también la elección de un nuevo presidente del Gobierno, la mayoría exigida en nuestra Constitución para derribar al anterior Gobierno permite una investidura automática de un nuevo presidente, por lo que no supone un bloqueo institucional, ya que tanto el antiguo como el nuevo Gobierno están garantizados, es decir, que favorece la pervivencia del antiguo mientras no prospere un nuevo Ejecutivo.

Por otro lado, resulta más interesante estudiar la posibilidad de bloqueo parlamentario que se ha vivido en la XI Legislatura pues, en el caso de no haberse presentado ningún candidato a la investidura, no se hubieran podido aplicar formalmente las previsiones del art. 99.5 CE para la disolución de las Cámaras y la convocatoria de las elecciones generales. Esto se debe a la exigencia de una mayoría parlamentaria, que es uno de los requisitos indispensables

3 La tramitación exige, al menos, el respaldo de la décima parte de los diputados que la presenten a la Mesa del Congreso, en escrito motivado en el que se incluya un candidato que haya aceptado esa postulación (art. 176.2 RC). Véase De Esteban y González-Trevijano (1994: 613). 
que marca el art. $99 \mathrm{CE}$, que se separa del procedimiento tradicional que se había seguido en nuestro constitucionalismo histórico, en el que lo habitual era la facultad enteramente libre del jefe del Estado para la designación del presidente del Gobierno.

En este sentido, la Constitución española de 1978 es una auténtica novedad en relación a esta cuestión, al situar la decisión en el seno del Congreso de los Diputados, que es realmente el órgano encargado de realizar la designación del nuevo presidente del Gobierno. La participación regia se va a situar en un contexto de unas atribuciones, más o menos formales, de nombramiento y de presentación del candidato o de los candidatos que cuenten con más posibilidades de obtener la confianza de la Cámara.

Una vez fijado que nuestro análisis se va a centrar en el denominado procedimiento ordinario de investidura del presidente del Gobierno, vamos a distinguir, dentro de las posibles causas que pueden originarlo, las siguientes:

a) El que se produce tras las elecciones generales.

b) El originado por la dimisión voluntaria del presidente del Gobierno.

c) El que se produce por su fallecimiento.

A estos supuestos habría que añadir, según De Esteban y González-Trevijano, otras dos circunstancias de carácter extraordinario que, si bien resultan poco usuales y difíciles en la práctica, no por eso se deben dejar de considerar y tener en cuenta.

En primer lugar, la posibilidad de un procesamiento y subsiguiente condena del presidente del Gobierno (art. 102 CE). En segundo lugar, el supuesto extraordinario que no se encuentra expresamente regulado en la Constitución y se daría en el caso de una enfermedad manifiesta que impida al presidente pedir su dimisión voluntaria (De Esteban y González-Trevijano, 1994: 277). A estos habría que añadir que los miembros del Gobierno pueden ser destituidos de los cargos públicos que ocupen por infracción muy grave (art. 26) de las recogidas en la Ley 3/2015, de 30 de marzo, reguladora del ejercicio del alto cargo de la Administración General del Estado.

Sin embargo, de todas estas posibilidades nos vamos a centrar básicamente en el proceso de elección del presidente del Gobierno ${ }^{4}$, pues nuestra Constitución diferencia, de una forma clara, el momento y el sistema de

4 En la doctrina, Ripollés Serrano (1980: 265-284) ha mantenido que resulta más preciso hablar de la «elección» del presidente del Gobierno por parte del Congreso de los Diputados en lugar de «nombramiento», sobre todo cuando la Cámara tiene que pronunciarse sobre un discurso de investidura. Por su parte, Bar Cendón (1983: 143) 
elección del presidente del de la formación del Gobierno propiamente dicho (Otto, 1980: 149). Además, debido a que, de todas estas alternativas, la que tiene mayor relieve es la elección del presidente puesto que, como muy acertadamente señala López Guerra (2013: 153), los procesos de formación y cese del Gobierno "giran esencialmente en torno a la figura del Presidente», cuyo nombramiento y cese es según este autor «el elemento definitorio, del que dependen el resto de los miembros del Gobierno».

\section{EL PAPEL DEL REY EN LA ELECCIÓN DEL PRESIDENTE DEL GOBIERNO}

Según el art. 1.3 CE la forma política del Estado español es la monarquía parlamentaria. De esta manera, nuestra Constitución parte de la configuración de la Corona como parlamentaria, lo que limita la prerrogativa regia para el libre nombramiento del presidente o de los ministros, a diferencia de otros textos de nuestro constitucionalismo histórico. No obstante, a pesar de encontrarse incardinada la designación del candidato a la presidencia del Gobierno a la consecución de la mayoría parlamentaria, supone, en la actualidad, una de las funciones más importantes asignadas constitucionalmente al monarca (Alzaga, 1978: 626).

En concreto, la propuesta realizada por el monarca de un candidato o de candidatos a la presidencia del Gobierno se produce, según el art. 99.1 CE, «después de cada renovación del Congreso de los Diputados y en los demás supuestos constitucionales en que así proceda». El mecanismo constitucional está regulado en el art. 99.1 CE, que atribuye al rey la facultad para consultar con los representantes designados por los grupos políticos, con representación parlamentaria, de forma posterior a las distintas renovaciones del Congreso de los Diputados, y en los demás supuestos recogidos en la Constitución, para proponer un candidato a la presidencia del Gobierno a través del presidente del Congreso de los Diputados.

En este sentido, hay que resaltar que la propuesta de candidatos a la presidencia del Gobierno la realiza el monarca «a través del Presidente del Congreso» (art. 99.1 CE), que entendemos que sería la persona en la que se apoyaría y asumiría la decisión del monarca ante cualquier problema que se pudiese originar, pues «la persona del Rey es inviolable y no está sujeta a responsabilidad» (art. 56.3 CE). En la doctrina Torres Muro ha puesto de relieve, precisamente, el relevante papel que desempeńa el presidente del Congreso en este proceso (Torres Muro 1995: 152).

mantiene que el proceso no consiste en «elegir» entre varios candidatos de una vez, sino que se debe votar a favor o en contra de aquel que se presenta en cada ocasión. 
En efecto, el rey es un símbolo de la unidad, árbitro y moderador del funcionamiento regular de las instituciones (art. 56.1 CE). Por tanto, la intervención regia se produce en un contexto de absoluta neutralidad política, que armoniza perfectamente con su configuración constitucional (art. 56.1 CE). En este contexto, el monarca debe buscar toda la información posible para realizar las consultas, como forma de no actuar desconociendo lo que sucede en la representación parlamentaria (Alzaga, 1978: 627), lo que será de gran relevancia en la decisión regia, para proponer aquel candidato o candidatos que más posibilidades tenga de salir investido por la Cámara baja.

En el derecho comparado, los procedimientos de elección del presidente del Gobierno son muy variados, y no coinciden exactamente con el recogido en nuestro texto constitucional, siendo el modelo alemán del art. 63 de la Ley Fundamental de la República Federal de Alemania de 1949 el más parecido al nuestro.

En Gran Bretańa el procedimiento está basado en la costumbre constitucional, según la cual la Corona puede nombrar a un primer ministro, que cuenta con la presunción del apoyo parlamentario, que se mantiene mientras no sea derribado por una votación en la Cámara.

Sin embargo, en Italia, según el art. 92 de la Constitución de 1947, el presidente de la República nombrará al presidente del Consejo de Ministros y, a propuesta de este, a los ministros. El precepto siguiente solo recoge que «dentro de los diez días siguientes a su formación, el Gobierno se presentará ante las Cámaras para obtener su confianza», puesto que según el mismo artículo «el Gobierno deberá gozar de la confianza de ambas Cámaras», pese a lo cual refuerza la estabilidad gubernamental al afirmar que «no comportará la obligación de dimitir el voto contrario de una de las Cámaras o de ambas sobre una propuesta del Gobierno». Por eso, hay autores que hablan de una doble investidura, en un proceso que permite la parlamentarización del nombramiento, pero en el que el presidente de la República sigue gozando de una preeminencia que pierde en otros sistemas (Torres Muro, 1995: 147).

En otras monarquías la intervención del jefe del Estado se reduce al mínimo. Este es el caso de Japón (art. 6 del texto constitucional de 1947) e, incluso, más limitado como en Suecia, donde el monarca no tiene capacidad ni de propuesta ni de nombramiento (arts. 2 a 4 del capítulo 6 del Instrumento de Gobierno de 1974).

En nuestro ordenamiento constitucional la doctrina ha mantenido que la propuesta regia de un candidato a la presidencia del Gobierno es un acto discrecional del rey. No obstante, Sánchez Agesta matiza que se debe entender efectivamente como una propuesta, pero no totalmente discrecional, pues está condicionada, en mayor o menor medida, por la información proporcionada 
por las consultas previas y, además, teniendo en cuenta la proporción que alcanza cada grupo parlamentario en el Congreso de los Diputados (Sánchez Agesta, 1991: 275).

Por otro lado, es verdad que el refrendo que se le exige al rey no supone más que un examen formal del acto regio ya que, según el mismo autor, «no tiene más alcance que una certificación de la corrección del acto», por ejemplo, que el monarca ha realizado las consultas previas que se exigen para tomar una decisión.

Sin embargo, la atribución regia de este tipo de cometidos resulta relevante, especialmente cuando ningún partido ha alcanzado la mayoría absoluta, o si el partido mayoritario no cuenta con los diputados suficientes para poder gobernar en minoría de una forma estable. Se trata de evitar cualquier responsabilidad del monarca trasladando esta al sujeto refrendante. No plantea grandes problemas cuando el candidato propuesto tiene garantizada la mayoría pues, entonces, el nombramiento del rey constituye formalmente «un acto absolutamente reglado» (González-Trevijano, 1998: 185).

No obstante, el problema es cuando no hay posibilidad de alcanzar la mayoría absoluta o al menos no existe un consenso parlamentario previo, reflexionando algunos autores como, entre otros, Aguiar, Garrorena, Revenga y Porras Ramírez, sobre una discreta labor de aproximación, por parte del rey, a las orientaciones de las fuerzas políticas. En este sentido, Vintró Castells (2016) entiende que, fundamentalmente, el monarca «debe proponer como primera opción al líder de la fuerza política con más escaños en el Congreso", pues, además, solo se comprueban los requisitos formales. Aunque el rey tiene la libertad de volver a proponer a un candidato que no hubiera conseguido una investidura previa, en primer lugar, porque la Constitución guarda silencio sobre este tema, en segundo lugar, porque no se puede descartar que un candidato pueda hacer propuestas programáticas alternativas, y que estas puedan lograr las mayorías necesarias. Por último, para este autor, «el Presidente del Congreso puede negar el refrendo a una propuesta de candidato, cuando apareciera como totalmente inviable derivar de la misma la investidura parlamentaria».

Según Péter Horváth, desde el punto de vista del derecho comparado no es habitual encontrar estudios especializados que analicen, de forma concreta, el papel desempeñado por el jefe del Estado en las consultas y en la presentación formal de candidatos para ocupar la presidencia del Gobierno. Este autor ha analizado este tema, en relación con la propuesta de candidatos efectuada por el presidente de la República en Hungría, llegando a la conclusión de que la intervención del presidente de la República, en aquel sistema constitucional, no tiene ninguna incidencia sobre el proceso político de formación del Gobierno, ni sobre la selección del primer ministro (Horváth, 2011: 22). 
Sin resultar tan tajantes se puede reflexionar sobre el hecho de que los monarcas, al igual que los presidentes de la República, suelen tener encomendadas unas funciones representativas, simbólicas y de neutralidad política que los suelen alejar de una intervención parcial en este tipo de negociaciones en las que pueden aflorar intereses partidistas.

Por otro lado, en el sistema constitucional español la intervención del rey tiene que estar en consonancia con la definición constitucional de la forma política del Estado español, como monarquía parlamentaria, habiéndose interpretado que el adjetivo "parlamentario» supone una translación del poder de decisión política del monarca al parlamento, tal y como sucede en otras monarquías europeas (Álvarez Conde y Tur Ausina, 2016: 580). De esta forma, la participación del rey en la propuesta de candidatos a la presidencia del Gobierno se sujeta en los principios de neutralidad política y respeto a las decisiones, pactos y acuerdos a los que pueden llegar las distintas fuerzas políticas, por lo que la convención será la de limitarse a proponer al candidato o candidatos que hayan logrado más apoyos en esas discretas negociaciones o que se estime que pueden conseguir las mayorías constitucionalmente requeridas.

Por otro lado, la práctica institucional de la Corona pone de relieve que, en ningún caso, en su participación de las distintas investiduras esta ha adoptado un papel de "protagonista material de la formación de los Gobiernos». Al contrario, hasta ahora las consultas han revestido «un carácter apenas protocolario y nunca han comprometido la neutralidad institucional de la Jefatura del Estado, cuyas propuestas al Presidente del Congreso han respondido siempre al designio de las negociaciones cerradas, entre el partido mayoritario y sus socios de legislatura o, sencillamente, a la lógica incontestable de la mayoría absoluta de un único partido» (Requejo Pagés, 2004: 80).

No obstante, si decidiese no sujetarse a este principio, no escrito, la votación parlamentaria se encargaría de demostrar si verdaderamente el candidato tiene o no la confianza de la Cámara, por lo que su intervención en este proceso no habría dado un resultado distinto de aquel que se quiere alcanzar en la votación parlamentaria. En cualquier caso, el buen juicio y sentido institucional del jefe del Estado le llevará a proponer a los candidatos que tengan más posibilidades de lograr la confianza de la Cámara en cada momento determinado, pues no debemos olvidar que se trata, como ya hemos dicho antes, de una monarquía parlamentaria (art. 1.3 CE).

En conclusión, «el Rey tiene de iure libertad de sugerir el candidato que estime conveniente. Pero en realidad no pasa de ser, en la mayoría de los casos, una posibilidad formal». Esto se debe a la caracterización de la monarquía como parlamentaria y a la necesidad del candidato propuesto por el rey de la 
confianza del Congreso de los Diputados. De esta manera, «no puede el Rey de facto pensar en respaldar un candidato que no pueda gozar al menos de mayoría simple en la Cámara baja. Luego el contenido de esta importante facultad regia está en última instancia condicionado por el sistema parlamentario y de partidos» (Alzaga et al., 2017: 297).

\section{LA SESIÓN DE INVESTIDURA: PERFILES CONSTITUCIONALES Y REGLAMENTARIOS}

En un sistema parlamentario una de las primeras y principales funciones del Parlamento es la de colaborar en la creación y en el mantenimiento de un Gobierno. Por eso, Bagehot (1966: 135) defendía que, aunque la Constitución no lo dijera expresamente, se entendía que se encomendaba al Parlamento británico la elección del Premier y su Gobierno.

En este contexto, la sesión de investidura es el acto parlamentario solemne en virtud del cual el Congreso de los Diputados delibera y decide, mediante discusión y votación, sobre la candidatura de un nuevo presidente y sobre el programa para la formación de su Gobierno.

Entre los requisitos previos necesarios que, según nuestro ordenamiento constitucional, son necesarios para que se pueda producir dicha sesión parlamentaria señalamos los siguientes:

a) La recepción de la propuesta del rey, que se publicará en el Boletín Oficial de las Cortes Generales.

b) La convocatoria del Pleno, según lo establecido el art. 170 del Reglamento del Congreso (RC).

c) La lectura de la propuesta que, según el art. 171.1 RC, se realizará por uno de los secretarios de la Cámara.

Cuando se cumplen estos requisitos el candidato procederá a exponer el programa político del Gobierno que pretende formar ante el Congreso de los Diputados, que es el órgano que tiene que otorgar su confianza, según lo dispuesto en el art. 99.2 CE. El Reglamento del Congreso no limita el tiempo de la exposición del candidato (art. 171.2 RC), si bien se espera que se ajuste a las normas, no escritas, del tiempo y de la cortesía parlamentaria.

En nuestra opinión, la discusión sobre el programa político del candidato supone uno de los momentos más importantes de la concesión de la confianza parlamentaria, puesto que el debate no se produce únicamente en torno a la elección del nuevo presidente del Gobierno, sino también sobre su programa 
gubernativo, cuyo voto favorable refuerza la relación fiduciaria que mantiene el Gobierno con el Parlamento.

La doctrina española mayoritariamente se pronuncia en el sentido de no reconocer efectos jurídicos al programa presentado en la sesión de investidura, pues considera que no condiciona las actuaciones del Gobierno ya investido (Gallego Anabitarte y Menéndez Rexach, 1985: 45).

En cualquier caso, el incumplimiento del programa, pese a no representar una responsabilidad jurídica, lo que podría implicar sería una responsabilidad política, por lo que un partido que hubiese obtenido su investidura sobre la base de dichas bases podría exponerse a una moción de censura o la pérdida de una votación de confianza.

En una línea parecida Revenga Sánchez reflexiona sobre la posible configuración de la investidura como un acto encuadrable en la función de control. Este autor recurre a la teoría de las funciones dentro del derecho parlamentario para situar la investidura en un contexto que «es el más característico de los actos por medio del cual la mayoría de la Cámara hace suya la orientación política global contenida en el programa de Gobierno», por lo que la investidura se podría configurar «a la vez como acto de control y como acto de participación en la función de dirección política» (Revenga Sánchez, 1988; 183).

En este sentido, se ha resaltado que las relaciones entre el Gobierno y el Parlamento tienen lugar entre dos instituciones autónomas, cada una con sus derechos y prerrogativas, que están atribuidos directamente por la misma Constitución, lo que pone de relieve la complejidad y relevancia de las relaciones entre los dos poderes del Estado (González-Trevijano, 1998: 4).

Precisamente, el debate de investidura es un filtro o test para que el Congreso se pueda pronunciar, tanto sobre las aptitudes del candidato para la formación de su equipo gubernamental como sobre los retos y la orientación del nuevo Gobierno. Por eso la discusión debe recoger las opiniones sobre las propuestas que va a llevar a cabo, que incluyan sus planes de actuación para el órgano Ejecutivo.

Por otro lado, la Ley del Gobierno 50/1997 no aclara el tema de cómo se debe presentar a la Cámara el Gobierno que se pretende formar. El art. 12 LG resulta en exceso lacónico, cuando remite el nombramiento y cese del presidente a «los términos previstos en la Constitución», utilizando también una fórmula breve, cuando en el apartado segundo del mismo artículo se establece el nombramiento y separación de los demás miembros del Gobierno, por el rey, a propuesta del presidente del Gobierno. Esta redacción resulta así idéntica a la empleada en el art. $100 \mathrm{CE}$.

Por tanto, la base sobre la que se asienta la investidura descansa en el art. 99 de la Constitución, que en sus apartados tercero y cuarto exige, para 
que la Cámara otorgue su confianza, la mayoría absoluta de sus miembros. En relación al desarrollo de la votación, el art. 99.2 CE prescribe que el candidato propuesto por el Rey «expondrá ante el Congreso de los Diputados el programa político del Gobierno que pretenda formar y solicita la confianza de la Cámara». También que, para el supuesto de no alcanzarse la mayoría absoluta, la misma propuesta se someterá a nueva votación, con un plazo de tiempo de 48 horas después de la anterior exigiéndose, en este caso, mayoría simple.

El supuesto previsto en la Constitución se produce cuando en ninguna de las dos votaciones se obtiene la investidura, por lo que el rey podrá presentar nuevas propuestas de candidatos, que se ajustarán a la forma descrita (art. 99.4 CE). No obstante, si no hay ningún candidato que logre la confianza del Congreso, en un plazo de dos meses, transcurridos a partir de la primera votación, el monarca procederá a disolver ambas Cámaras y convocar nuevas elecciones, con el refrendo del presidente del Congreso (art. 99.5 CE).

La previsión de disolución de las Cámaras, después de trascurridos dos meses de la primera votación, supone, para De Esteban y González-Trevijano, una auténtica cláusula de cierre del sistema de nombramiento del presidente tomada, en parte, del art. 3. ${ }^{\circ}$ del capítulo VI de la Constitución sueca y, por otro lado, del art. 63 de la Ley Fundamental de Bonn. Se trataría de una "especie de aviso" para proceder a la formación de un Gobierno, lo antes posible, con el objetivo de que, al menos, pueda durar un año, que es el plazo de tiempo que se debe esperar, como mínimo, para una nueva disolución de las Cámaras legislativas, según el art. 115.3 CE.

En otros ordenamientos existen previsiones constitucionales similares, como en el caso de la Ley Fundamental de Hungría de 2011, que en su art. 16.4 establece que el primer ministro será elegido con los votos de más de la mitad de los miembros de la Asamblea Nacional, y también que, para el supuesto en que el Parlamento no elija ningún primer ministro, después de cuarenta días de la primera nominación por el presidente de la República, que este último pueda disolver el Parlamento a su propia discreción (Horváth, 2011: 6).

En Espańa, el procedimiento cuando un candidato obtiene la confianza de la Cámara, mediante mayoría absoluta en la primera votación o mayoría simple en la segunda ${ }^{5}$, es que el presidente del Congreso transmita, de forma

Para la consecución de estas mayorías no tiene ninguna relevancia que puedan existir escaños vacantes, debido a cualquier problema o a contenciosos electorales pendientes, para alcanzar la llamada "condición plena de diputado". Por otro lado, el art. 164.1 de la Ley 5/1985, de 19 de junio, del Régimen Electoral General manifiesta que: «en caso de fallecimiento, incapacidad o renuncia de un diputado, el escaño será 
inmediata, la voluntad expresada por la Cámara al rey (arts. 99.3 y 62 d) CE), que, de conformidad con nuestra tradición histórica, asume el acto del Congreso de los Diputados mediante la forma de Real Decreto.

El nombramiento se considera un acto típico de la Jefatura estatal, dotado de automatismo y encuadrable dentro de las funciones que se atribuyen al rey, como símbolo de la unidad y permanencia del Estado. El refrendo de este acto corresponde al presidente del Congreso (art. 64.1), eliminando el problema de que el primer ministro saliente deba responsabilizarse de la designación de un posible adversario político (González-Trevijano, 1998: 181).

En conclusión, entendemos que lo más relevante de la configuración constitucional del art. $99 \mathrm{CE}$ es la importancia que atribuye al Congreso de los Diputados en sus distintos requerimientos y exigencias para la investidura del presidente del Gobierno, tales como la consulta previa del rey, con los representantes designados por los grupos políticos presentes en la Cámara, la exposición del programa político del Gobierno que desea formar el candidato, y la consecución de las mayorías necesarias para que la Cámara exprese la obtención de su confianza.

En este sentido, el criterio de la mayoría absoluta revela el deseo del constituyente de propiciar un Gobierno fuerte, con una amplia base o respaldo parlamentario, que dote de estabilidad la labor ejecutiva. No obstante, la importancia de favorecer, lo antes posible, la formación del Gobierno aconseja rebajar dicho apoyo en una mayoría simple, pues la falta de acuerdo en la formación de un nuevo Gobierno no incide, solo, sobre el desarrollo de sus normales funciones y atribuciones, sino que afecta, entre otras transcendentales cuestiones, a otros órganos e instituciones del Estado, que mantienen grandes e importantes relaciones con el órgano Ejecutivo.

\section{LAS DIFERENTES FORMAS DE INVESTIDURA EN LOS ESTATUTOS DE AUTONOMÍA}

Los estatutos de autonomía de la mayoría de las comunidades autónomas siguen, con mayor o menor fidelidad, la fórmula recogida en el art. 99 CE. En este sentido, Péndas García (1988: 86) ha puesto de relieve la falta de «imaginación política» de ciertos estatutos, y la oportunidad perdida para haber repetido algunas «fórmulas superadas por su manifiesta inadecuación a la realidad social».

atribuido al candidato o, en su caso, al suplente, de la misma lista a quien corresponda, atendiendo a su orden de colocación». Véase Revenga Sánchez (1988: 217). 
Sin embargo, es posible señalar algunos estatutos que han propuesto alguna variación o alternativa a esta regulación para que, en los requisitos y los procedimientos para la formación del Gobierno de la comunidad autónoma, se pudiesen tratar los problemas que se han vivido en la XI legislatura del Parlamento nacional. Sin olvidar el problema surgido en la Comunidad de Madrid, en las elecciones autonómicas de 2003, que originó el dictamen del Consejo de Estado de 2003, que consideró que la ausencia de candidatos «es equivalente a una votación fallida», empezando a contar el plazo desde que se constata la imposibilidad de proponer un candidato, lo que debemos interpretar como un intento de este órgano de buscar algún tipo de solución para salir de una situación tan grave y complicada ${ }^{6}$.

Sin embargo, el sistema utilizado en esta comunidad, para repetir las elecciones autonómicas, no se podría aplicar al Parlamento nacional, pues el art. 182 del Reglamento de la Asamblea Regional de Madrid fija un plazo de 15 días para que se presente un candidato, y si no existe esa presentación se inicia la cuenta atrás, que dura dos meses, que es el plazo prescriptivo para poder convocar unos nuevos comicios en esta comunidad ${ }^{7}$.

El problema es que ni en la Constitución española, ni en los reglamentos parlamentarios, del Congreso ni del Senado, existe ese plazo inicial, ni tampoco, por lo tanto, se contempla que no se presente ningún candidato a la presidencia del Gobierno nacional. Por eso, el Gobierno en funciones manifestó, en su día, la preocupación por la situación de bloqueo en la anterior legislatura nacional, antes de la presentación de la candidatura socialista, pues a pesar del posible precedente, en última instancia, del citado dictamen del Consejo de Estado, la vicepresidenta en funciones, Soraya Saénz de Santamaría, reconoció públicamente que existía un preocupante «vacío constitucional» acerca de si se pueden activar los plazos para ir a unas nuevas elecciones si no hay un candidato claro para una sesión de investidura ${ }^{8}$.

Por tanto, será conveniente buscar otras fórmulas jurídicas para superar estas dificultades, y algunos estatutos de autonomía han propuesto distintas soluciones con mayor seguridad jurídica, entre los que podemos destacar el

6 Véase el dictamen del Consejo de Estado: http://www.boe.es/buscar/doc.php?id=CE-D-2003-1985.

7 Véase el art. 182 del Reglamento de la Asamblea de Madrid, actualizada a 21 de febrero de 2017.

8 Véase el artículo: «La repetición de elecciones en Madrid tras el tamayazo no sirve de modelo para disolver las Cortes sin investidura». Disponible en: http://www.europapress.es/nacional/noticia-repeticion-elecciones-madrid-tamayazo-no-sirve-modelo-disolver-cortes-investidura-20160129173808.html. 
Estatuto de Autonomía para Asturias, que prevé que «si transcurrido el plazo de dos meses a partir de la constitución de la Junta, ningún candidato hubiera sido elegido, la Junta General electa quedará disuelta, procediéndose a la convocatoria de nuevas elecciones»?. De esta forma, el plazo empieza a contar desde la constitución de la Junta General del Principado de Asturias, que es el órgano legislativo de la comunidad autónoma, pero no se vincula expresamente a una primera votación o a la constatación de que no se va a presentar algún candidato a la investidura.

Lo mismo se puede decir del Estatuto de Autonomía de Aragón, que fija el plazo de dos meses, a partir de la constitución de las Cortes de Aragón, para el supuesto en el que ningún candidato hubiese sido investido, por lo que el plazo no cuenta desde la presentación de un candidato, sino desde los dos meses de la constitución del órgano legislativo, quedando en ambos casos las Cortes de dicha comunidad autónoma disueltas y convocándose nuevas elecciones ${ }^{10}$.

En el Estatuto de Autonomía de Castilla-La Mancha el presidente de la Junta de Comunidades se elige también por mayoría absoluta de las Cortes autonómicas, y por mayoría simple a las cuarenta y ocho horas después de la anterior votación. Sin embargo, para el caso de no alcanzarse esta mayoría, y después de haberse tramitado «sin debate sucesivas propuestas y si en ninguna de ellas se llegara, en el plazo de dos meses, a alcanzar la mayoría simple, quedará automáticamente designado el candidato del partido que tenga mayor número de escaños» ${ }^{11}$.

En este supuesto se resuelve, de forma aparentemente sencilla, la falta de acuerdos o consensos necesarios para la obtención de una mayoría suficientemente significativa en la Cámara, para proceder a la formación del Gobierno autonómico. Sin embargo, en relación a esta propuesta hay que matizar que a esta solución se debe llegar solo en el caso de que no sea posible la formación de un Gobierno con el máximo respaldo parlamentario, al encontrarnos lógicamente en un sistema parlamentario. En efecto, los Gobiernos en minoría parlamentaria se encuentran más limitados para llevar una actividad legislativa y gubernamental más amplia, siendo necesarios los acuerdos puntuales para sacar adelante iniciativas legislativas o políticas de mayor complejidad o

9 Art. 32 de la Ley Orgánica 7/1981, de 30 de diciembre, de Estatuto de Autonomía para Asturias.

10 Art. 48. Investidura. Ley Orgánica 5/2007, de 20 de abril, de reforma del Estatuto de Autonomía de Aragón.

11 Art. 14 de la Ley Orgánica 9/1982, de 10 de agosto, de Estatuto de Autonomía de Castilla-La Mancha. 
importancia. Por esta razón, se deben explorar, previamente al acto de investidura, todas las opciones políticas que supongan un mayor respaldo y apoyo a la labor del órgano Ejecutivo por parte del Parlamento autonómico, por lo que tomamos con mucha precaución la formación automática o mecánica de los Gobiernos autonómicos.

Algo similar recoge la regulación contenida en el art. 29 de la normativa relativa a este tema de la Comunidad Foral de Navarra, que establece un plazo de tres meses, desde la celebración de las elecciones al Parlamento de Navarra, a partir del que se empieza a contar, tanto en el supuesto de que «no se presentara ningún candidato o ninguno de los presentados hubiera sido elegido» para que, tanto en uno como en otro supuesto, se disuelva el Parlamento y se convoquen de inmediato nuevas elecciones, con la salvedad de que «el nuevo Parlamento que resulte de la convocatoria electoral tendrá un mandato completo por un período de cuatro años» ${ }^{12}$.

Este artículo fue reformado y ha quedado redactado de la siguiente forma: «Si transcurrido el plazo de dos meses a partir de la primera votación ningún candidato hubiera resultado investido, el Presidente del Parlamento propondrá al Rey el nombramiento del candidato que designe el partido político, federación de partidos, agrupación o coalición electoral que cuente con mayor número de escaños. En caso de empate en el número de escaños, el candidato será designado por el partido, federación de partidos, agrupación o coalición electoral, cuya lista hubiera obtenido el mayor número de votos» ${ }^{13}$.

Desde un punto de vista práctico, las facilidades para evitar posibles bloqueos políticos en esta regulación autonómica son evidentes. No obstante, en nuestra opinión, el sistema parlamentario se debe basar en la búsqueda de un

12 Art. 29 de la Ley Orgánica 13/1982, de 10 de agosto, de reintegración y amejoramiento del Régimen Foral de Navarra (LORAFNA). El texto original era de 1982, pero en la última actualización de 2010 se dice que «si trascurrido el plazo de tres meses desde la celebración de las elecciones al Parlamento de Navarra no se presentara ningún candidato o ninguno de los presentados hubiera sido elegido, el Parlamento quedará disuelto, convocándose de inmediato nuevas elecciones». Véase el fallo de la STC 16/1984, de 6 de febrero de 1984, a raíz de la interpretación que se hizo de esta cuestión tras las elecciones al Parlamento de Navarra del 8-5-1983. En el fundamento jurídico 8 de la STC 15/2000 se dice que «en el procedimiento extraordinario la designación de Presidente de la Diputación Foral ha de recaer en el candidato que haya sido presentado formalmente a la Presidencia del Parlamento por el grupo político que hubiera obtenido en las elecciones el mayor número de escaños o, en caso de empate, el mayor número de votos».

13 Ley Foral 9/1991, de 16 de marzo (BON de 25 de marzo de 1991; BOE de 20 de septiembre de 1991). 
equilibrio y respeto a las formaciones políticas minoritarias, por lo que, lógicamente, la regulación foral prefiere, en un primer intento, las soluciones consensuadas para proponer candidatos que puedan lograr la confianza de la Cámara, y solo en caso de que ningún candidato hubiera resultado investido, se pueda proponer al candidato con mayor número de escańos. Que en la norma se utilizase, en primer lugar, el criterio de los escaños y no el de mayor número de votos pone, precisamente, de relieve que el presidente del Gobierno autonómico debe ser aquel que mayor apoyo concita en el órgano legislativo, pues, recordemos, la confianza para gobernar, en el sistema parlamentario, la confieren las mayorías parlamentarias. Solamente pare el caso de empate en el número de escaños se puede recurrir al criterio de la fuerza que haya obtenido el mayor número de votos, circunstancia que debe ser, en buena lógica, el último recurso en ser utilizado, por las razones expuestas.

Por tanto, el procedimiento de votación para la elección del presidente varía en cada comunidad autónoma. Sin embargo, la mayor parte sigue el modelo establecido en el art. 99 CE. Esto supone dos grandes ideas: la exigencia de mayoría absoluta en una primera votación y el fijar (salvo los estatutos gallego y valenciano) instrumentos de racionalización que no dilaten sin fin el proceso de elección (Bastida Freijedo, 2003: 559)

Un caso especial es la elección del presidente de Ceuta y de Melilla. En la elección del presidente de Ceuta la votación se produce, por mayoría absoluta, entre los miembros de la Asamblea de Ceuta, siendo sus integrantes los que encabezaran alguna de las listas electorales que hayan obtenido escaño. Para el caso de que ningún candidato hubiese logrado dicha mayoría «quedará designado Presidente el que encabece la lista que hubiera obtenido mayor número de votos» ${ }^{14}$.

De forma similar, el presidente de Melilla, que también ostenta la condición de alcalde de la ciudad, es elegido por parte de la Asamblea de Melilla por mayoría absoluta e, igualmente, si ningún candidato obtiene dicha mayoría, quedará designado presidente quien encabece la lista con mayor número de votos ${ }^{15}$.

Para empezar, hay que resaltar que lo primero que se propone es la mayoría absoluta de los miembros de la Asamblea, por lo que se prima la búsqueda de una solución que permita contar con la confianza de la Asamblea, y solamente en caso de no poderse alcanzar esta se recurre al criterio de la lista que cuente con mayor número de votos.

14 Art. 15 de la Ley Orgánica 1/1995, de 13 de marzo, de Estatuto de Autonomía de Ceuta.

15 Art. 15 de la Ley Orgánica 2/1995, de 13 de marzo, de Estatuto de Autonomía de Melilla. 
En nuestra opinión, esta fórmula de recurrir al número de votos, en caso de no conseguirse la mayoría absoluta, no sería exportable ni a los Gobiernos de las comunidades autónomas ni a las instituciones del Estado, puesto que, como ya hemos dicho, nuestro sistema de gobierno se basa en el modelo de parlamentarismo que se caracteriza, entre otras razones, por la estrecha y fructífera relación de confianza entre el órgano ejecutivo y el parlamentario y, además, este es un modelo adaptado a las peculiaridades intrínsecas de estas dos ciudades autónomas.

\section{LA PROPUESTA DE REFORMA CONSTITUCIONAL DEL ART. 99.5 CE}

Las duodécimas elecciones generales en España, desde la transición a la democracia, se celebraron el día 20 de diciembre de $2015^{16}$, con un resultado electoral que no permitió a ningún partido obtener los suficientes escaños en el Congreso de los Diputados como para superar la mayoría absoluta, necesaria para la primera votación en la Cámara exigida por el art. 99 CE.

De esta forma, se hace obligatorio llegar a pactos para intentar alcanzar la mayoría simple en una segunda votación. No obstante, los datos de la representación alcanzada por los distintos partidos políticos, en la Cámara Baja, hacen muy complicado la suma de las fuerzas suficientes de los partidos políticos, de ideologías afines, en el arco izquierda-derecha, como para que la investidura de algún candidato a presidente del Gobierno pueda tener ciertas garantías de éxito ${ }^{17}$.

Después de una sesión de investidura fallida, en la que el Partido Socialista Obrero Español (PSOE) presenta a Pedro Sánchez como candidato a la presidencia del Gobierno no logra los suficientes apoyos como para la formación de Gobierno ${ }^{18}$, el rey disuelve las Cámaras, con el refrendo del Presidente del Congreso de los Diputados, mediante el Real Decreto 184/2016, de 3 de mayo, de disolución del Congreso de los Diputados y del Senado. En el mismo

16 Real Decreto 977/2015, de 26 de octubre, de disolución del Congreso de los Diputados y del Senado y de convocatoria de elecciones. Boletín Oficial del Estado, núm. 257, de 27-X-2015, sec. I, p. 100784.

17 Los datos del Ministerio del Interior de las elecciones generales del 20-12-2015 se pueden consultar en: http://www.infoelectoral.interior.es/min/busquedaAvanzadaAction.html

18 Los datos del debate de investidura, que empezó el 1-3-2016, en: Diario de sesiones del Congreso de los Diputados. Pleno y Diputación Permanente, año 2016, XI Legislatura, núm. 3, p. 104. 
real decreto se convocan las elecciones generales, para el domingo 26 de junio de 2016 (art. 2), y se fijan las sesiones constitutivas de las Cortes para el día 19 de julio de 2016 (art. 5) 19

La novedad de esta sesión de investidura es que se trata de la primera vez, en la historia de la democracia espańola, que el candidato que propone el rey no obtiene la confianza de la Cámara para formar Gobierno, si bien la presentación de este candidato por el rey permite resolver el problema que plantea el art. 99.5 CE en relación a la obligatoriedad de celebrar una sesión de investidura para que el plazo de dos meses pueda contabilizarse.

En efecto, la única salida posible a la situación descrita, desde el punto de vista constitucional, es la disolución de las Cámaras y la convocatoria de unas nuevas elecciones generales, que se celebran el 26 de junio. En estos comicios se produce un incremento de 14 diputados del Partido Popular en relación a las anteriores elecciones de 2015, pero lo sitúan todavía a gran distancia de la mayoría absoluta y hacen inevitables los pactos o abstenciones para que gobierne solo un partido, sin algún tipo de acuerdo, es decir, como la fuerza política con más representación parlamentaria unitaria no debida a acuerdos o a pactos poselectorales ${ }^{20}$.

Finalmente, gracias a las negociaciones y a los pactos poselectorales se produce la investidura ${ }^{21}$ de Mariano Rajoy como presidente del Gobierno, el 29 de octubre de 2016. Los datos de la votación se recogen en el Real Decreto 414/2016, de 30 de octubre, en el que se plasma la confianza otorgada por el Congreso de los Diputados, por mayoría simple, en una segunda votación, que se celebró el 29, de acuerdo con lo establecido en el apartado tres del art. 99 CE.

El deseo de superar los problemas de la anterior legislatura se encuentra implícito en el discurso del candidato a la presidencia del Gobierno, que, por otro lado, se esfuerza en afirmar que «Espańa puede ser gobernada, y bien gobernada, aunque no exista mayoría absoluta» y, en este sentido, pide los apoyos parlamentarios para desarrollar las tareas del futuro Gobierno.

19 Real Decreto 184/2016, de 3 de mayo, de disolución del Congreso de los Diputados y del Senado y de convocatoria de elecciones. Boletín Oficial del Estado, núm. 107, 3-V-2016, sec. I, p. 29959.

20 Los datos del Ministerio del Interior de las elecciones generales del 26-6-2016 se pueden consultar en: http://resultados2016.infoelecciones.es/99CO/DCO99999XX. htm?lang=es

21 En la sesión del 29 de octubre de 2016, el Pleno del Congreso de los Diputados otorgó a Mariano Rajoy su confianza por 170 votos a favor, 111 en contra y 68 abstenciones. Véase el Diario de Sesiones del Congreso de los Diputados. Pleno y Diputación Permanente, XII Legislatura, núm. 14, de 29 de octubre de 2016, p. 22. 
La investidura de Rajoy pone fin al periodo más largo de inestabilidad política de la democracia española, que se materializa en diez meses del Gobierno en funciones de Mariano Rajoy. Las negociaciones para salir de esta situación fueron complejas, y se ponen en evidencia en el hecho de que fuese precisa la abstención de 68 de los 85 diputados socialistas, además de un pacto, suscrito entre el Partido Popular y Ciudadanos, denominado «150 compromisos para mejorar Espańa», firmado el 28 de agosto de $2016^{22}$.

En la actualidad, se cuestiona desde algunos sectores políticos la posibilidad de llevar a cabo algunas de las medidas del pacto. No obstante, a pesar de los problemas para un buen entendimiento entre las distintas fuerzas políticas pensamos que los problemas y dificultades de la XI Legislatura, que se cerró con la imposibilidad de constituir un Gobierno, han supuesto un acicate para una mayor concienciación sobre la necesidad de que se superen en el Parlamento posibles bloqueos institucionales como los del anterior período de sesiones.

En este sentido, partimos de que los partidos políticos cumplen una valiosa función de dar cauce, manifestar y defender la rica diversidad de opiniones y pensamiento de un amplio electorado que necesita ser representado, pero que también se les puede demandar que sean conscientes de los problemas generales, tanto de los ciudadanos como del Estado, tratando de conjugar, como resulta habitual en la política y en el derecho comparado, los intereses particulares con los generales.

Por tanto, apelando al buen juicio y a la razón de Estado de todos los partidos políticos, debemos sacar consecuencias y aprender de lo ocurrido en la anterior legislatura. Por eso, en nuestra opinión, que resulte deseable una buena articulación de los intereses particulares con los generales, por las distintas fuerzas políticas, no representa ningún impedimento para proponer, además, una modificación del párrafo quinto del art. $99 \mathrm{CE}$ que evite el posible bloqueo institucional que puede producirse en el caso de nadie se postule como candidato a la presidencia del Gobierno, por ejemplo, al no contar realmente con los suficientes apoyos parlamentarios, pues a nadie se le puede exigir que presente su candidatura, y menos aún cuando no tiene el respaldo de los escaños suficientes para respaldar su propuesta.

Por eso, entendemos que no resulta conveniente seguir manteniendo una regulación que parte de una presunción en la que, al menos, un candidato a la presidencia del Gobierno se haya tenido que someter a la sesión de investidura. De esta forma, no ha sido prevista la posibilidad de que nadie

22 Los «150 compromisos para mejorar España» pueden consultarse en: https://www. okdiario.com/img/2016/08/28/150compromisos.pdf (último acceso el 10-3-2016). 
se presente a esa primera votación de investidura debido, básicamente, a no contar con el suficiente respaldo parlamentario, con lo que el cómputo del plazo para la disolución de las Cámaras no empezaría a contar nunca, y la situación de bloqueo político e institucional que se crearía podría terminar afectando a las principales instituciones del Estado, debido al vacío o a la falta de previsión de este tipo de supuesto, con lo que se haría inevitable recurrir al citado Dictamen del Consejo de Estado como única alternativa para salir de este obstáculo.

Sin embargo, esta posibilidad no resulta tan remota como podría pensarse, pues la idea del parlamentarismo como modelo que propicia la discusión, debate y decisiones de los diputados está dando, cada día, mayor relevancia a las estrategias y decisiones partidistas. En este sentido, como ha dicho Aragón Reyes, el verdadero protagonismo en la actividad de las Cámaras se asienta en los partidos, y no en los parlamentarios individuales. Por eso, se puede hablar de que hoy en día es el Gobierno el que dirige a la mayoría parlamentaria, «invirtiéndose la relación de subordinación, hasta el punto de que ha podido decirse que en la actualidad es el Parlamento el comité legislativo del Gobierno. Por todo ello, la posibilidad de que triunfe una moción parlamentaria de censura es bastante remota y, en consecuencia, la responsabilidad política del Gobierno parece más una proclamación retórica que una regla efectiva» (Aragón Reyes, 2005).

Precisamente debido a esta configuración, en ocasiones se pueden plantear retos y dificultades que hagan muy complicada o ardua la tarea de entendimiento entre los partidos, lo que puede terminar incidiendo sobre la investidura del presidente del Gobierno. Por eso, en nuestra opinión, con el suficiente consenso y tranquilidad necesarias, en el momento adecuado, se podría abordar este espinoso problema. Nuestra propuesta apuesta por una reforma constitucional del art. 99.5 CE que busque soluciones de una forma análoga a las que, en el epígrafe anterior, hemos visto que se han propuesto en algunos estatutos de autonomía españoles. En concreto, la reforma que proponemos del citado precepto quedaría de la siguiente forma:

Art. 99.5 CE: «Si transcurrido el plazo de dos meses, a partir de la constitución del Congreso de los Diputados, sin que se haya presentado ningún candidato a la Presidencia del Gobierno o en el caso de transcurrido el plazo de dos meses, a partir de la primera votación de investidura, sin que ningún candidato hubiere obtenido la confianza del Congreso, el Rey disolverá ambas Cámaras y convocará nuevas elecciones con el refrendo del Presidente del Congreso».

En nuestra opinión, la presunción de los pactos y acuerdos entre las fuerzas políticas con representación en el Congreso de los Diputados no debe 
considerarse una premisa tácita incuestionable, pues, a pesar del carácter parlamentario de nuestro sistema de gobierno, no se debe desconocer que el ejercicio y los equilibrios del poder son a veces de una geometría muy compleja y variable. Por eso, la modificación que proponemos en la redacción del nuevo art. 99.5 CE pensamos que resulta perfectamente compresible y pertinente por las razones aducidas.

\section{CONCLUSIONES}

Una vez estudiado y analizado el significado y alcance de la sesión de investidura del candidato a la presidencia del Gobierno, como factor de colaboración entre las instituciones, de aceptación expresa de un programa de gobierno y de estabilidad política e institucional, exponemos de forma breve las aportaciones más importantes de nuestro trabajo.

1. En primer lugar, mantenemos que la sesión de investidura, en nuestro país, no es solo un mero trámite o procedimiento parlamentario celebrado en el Congreso de los Diputados, sino en realidad un verdadero filtro o test que pone de relieve las opciones reales que tiene un candidato a la presidencia del Gobierno para la formación de un equipo de Gobierno, para implementar sus futuras líneas de actuación y para su propia estabilidad mientras dure su mandato, pues le permite formular y exponer los principios básicos sobre los que se va a desarrollar su labor gubernamental y la relación que previsiblemente se mantendrá entre el Ejecutivo y el Legislativo a lo largo de la legislatura.

2. En segundo lugar, constatamos que el procedimiento formulado en el art. $99 \mathrm{CE}$ se inserta dentro del clásico parlamentarismo racionalizado, dotado de aquellos instrumentos y medios que aseguran la concesión de la confianza del Congreso de los Diputados al órgano Ejecutivo, en el inicio de lo que debería ser una larga y fructífera relación de cooperación entre la mayoría parlamentaria, que lo apoya, y el Gobierno. Por tanto, el acento no se debe poner solo en la consecución de una determinada mayoría, sino si este tipo de confianza y respaldo se formulan desde premisas sólidas que permitan que la legislatura pueda agotar su mandato constitucional de forma pacífica y productiva, lo que produce, también, una estabilidad política e institucional.

3. En tercer lugar, afirmamos que la participación del rey, a través de la propuesta de candidato o candidatos, previa consulta con los representantes 
designados por los grupos políticos con representación parlamentaria, y su nombramiento o la disolución de las Cámaras, prevista en el art. 99.5 $\mathrm{CE}$, que se encuentra en ambos casos bajo el refrendo del presidente del Congreso (art. 64.1 CE), resulta, en general, similar a la que mantienen otros jefes del Estado, tanto en monarquías como en repúblicas. Las características intrínsecas de esta alta magistratura, como símbolo de la unidad, árbitro y moderador del funcionamiento regular de las instituciones (art. 56.1 CE), le confieren un carácter neutro, separado de cualquier decisión política que, opinamos, resulta acorde con su configuración constitucional, lo cual se inserta también en un contexto parecido en muchos de los modelos de Jefatura del Estado del derecho constitucional comparado.

4. En cuarto lugar, ponemos de relieve que en nuestro sistema constitucional la previa investidura del presidente del Gobierno, por el Congreso de los Diputados, resulta imprescindible para poder constituir un Ejecutivo, de modo que solo tras la obtención de la confianza parlamentaria del presidente se pueden dictar los decretos de nombramiento de los ministros y del resto del equipo gubernamental, una vez que se ha constatado, expresamente, que se ha obtenido la confianza de la Cámara Baja para gobernar. Por tanto, nuestro modelo es indiscutiblemente de formación parlamentaria «explícita» del Gobierno, en la línea de lo establecido por otras Cartas Magnas, como la Constitución francesa de 1946, la Ley Fundamental de Bonn de 1949, la Constitución japonesa de 1946 o la sueca de 1974. En consecuencia, en nuestro régimen constitucional la investidura del presidente del Gobierno es una fase imprescindible y necesaria para la formación del Gobierno, que se justifica por las razones que hemos analizado a lo largo de este trabajo de investigación.

5. Hemos analizado en este trabajo la normativa autonómica, especialmente aquella que ha propuesto alguna solución al bloqueo institucional, cuando ningún candidato a la presidencia del Gobierno autonómico consigue el suficiente apoyo parlamentario, en el plazo de dos meses. En alguno de esos estatutos de autonomía que hemos estudiado se recoge que, en ese caso, "quedará automáticamente designado el candidato del partido que tenga mayor número de escańos». Hemos formulado una matización a esta regulación, pues entendemos que se trata de un caso extremo al que se debe llegar solo cuando no sea posible la formación de un Gobierno con un amplio respaldo parlamentario, al encontrarnos lógicamente en un sistema parlamentario, configurado sobre la base de la más amplia confianza 
posible, para que el órgano ejecutivo tenga la mayor estabilidad, siendo el Gobierno en minoría la última de las opciones y, en general, la menos deseable en términos de estabilidad e interés general.

6. Por otro lado, consideramos que no se puede utilizar la fórmula de recurrir a la lista que hubiera obtenido mayor número de votos cuando no se pueda alcanzar la mayoría absoluta de la Cámara, como en el caso de las ciudades autónomas de Ceuta y Melilla, en un ámbito territorial y político distinto al de estas dos singulares ciudades, debido, como ya hemos explicado, a las peculiaridades que adopta esta configuración inserta en aquellas características particulares, también, de su organización local.

7. Por último, deseamos resaltar la importancia de la colaboración y del libre juego de las fuerzas políticas para lograr la investidura del presidente del Gobierno, en el contexto del modelo del parlamentarismo. Sin embargo, para prevenir posibles situaciones de obstrucción y bloqueo político proponemos una reforma de la Constitución, en su art. 99.5 $\mathrm{CE}$, para que el rey pueda disolver las Cámaras y convocar elecciones, incluso, si no se presenta ningún candidato, pues entendemos que en democracia no se puede forzar a las fuerzas políticas a proponer candidaturas ni a sus líderes a presentarse como candidatos, sobre todo cuando no se cuenta con el apoyo parlamentario necesario. Por eso, mantenemos que se debe adoptar una previsión constitucional que permita, también cuando no se presentan candidatos a la presidencia del Gobierno, disolver las Cámaras y recurrir, en última instancia, al electorado, pues del pueblo español «emanan los poderes del Estado» (art. 1.2 CE), por lo que resulta perfectamente plausible que sean los ciudadanos los que decidan, nuevamente, en los comicios generales cómo resolver estas posibles situaciones de obstrucción política e institucional, como uno de los mecanismos habituales propios del sistema democrático.

\section{Bibliografía}

Álvarez Conde, E. y Tur Ausina, R. (2016). Derecho constitucional. Madrid: Tecnos.

Alzaga, O. (1978). La Constitución española de 1978: Comentario sistemático. Madrid: Foro.

— - Gutiérrez Gutiérrez, I., Reviriego Picón, F. y Salvador Martínez, M. (2017). Derecho politico español según la Constitución de 1978. T. II: Derechos fundamentales y Órganos del Estado. Madrid: Ramón Areces.

Aragón Reyes, M. (2005). Gobierno y forma de gobierno: problemas actuales. En M. Aragón Reyes y A. Gómez Montoro (coords.). El Gobierno. Problemas constitucionales (pp. 27-66). Madrid: CEPC. 
Bagehot, W. (1966). The English Constitutions. London: Collins.

Bar Cendón, A. (1983). El presidente del Gobierno en España. Encuadre constitucional y práctica política. Madrid: Civitas.

Bastida Freijedo, F. (2003). El presidente del Principado. En L. Arce Janáriz (coord.). El Estatuto de Autonomía del Principado de Asturias. Un estudio sistemático. Oviedo: Junta General del Principado de Asturias.

De Esteban, J. y González-Trevijano, P. (1994). Curso de Derecho constitucional español. Vol. III. Madrid: UCM.

De Winter, L. (1995). The role of parliament in government formation and resignation. En H. Doring. Parliaments and majority rule in Western Europe. Disponible en: http://allman.rhon.itam.mx/-emagar/ep3/rules/doring.ed.parliamentsAndMajRule1995. pdf\#page $=115$.

Gallego Anabitarte, A. y Menéndez Rexach, A. (1985). Artículo 97. Funciones del Gobierno. En O. Alzaga. Comentarios a las Leyes Politicas. Constitución Española de 1978. Madrid: Edersa.

González-Trevijano, P. (1998). El refrendo. Madrid: CEPC.

Horváth, P. (2011). The investiture vote in Hungary. Legislative Power and the Role of Investiture in Parliament, at the 6th General Conference of the European Consortium for Political Research, Reykjavik, 25th-27th August 2011.

López Guerra, L., Espin Templado, E. y García Morillo, J. (2013). Derecho constitucional (vol. II): los poderes del Estado: organización territorial del Estado. Valencia: Tirant lo Blanch.

Martin, S. (2011). The Design and Consequences of Investiture Rules: The Irish Case. Legislative Power and the Role of Investiture in Parliament, at the 6th General Conference of the European Consortium for Political Research, Reykjavik, 25th-27th August 2011.

Mateos y de Cabo, O. I. (2006). El Presidente del Gobierno en España: status y funciones. Madrid: La Ley.

Mellado Prado, P. (1988). La responsabilidad politica del Gobierno en el ordenamiento español. Madrid: Congreso de los Diputados.

Montero Gibert, J. R. (1984). Moción de censura. En J. J. González Encinar. Diccionario del sistema político español. Madrid: Akal.

Otto y Pardo, I. (1980). La posición constitucional del Gobierno. Documentación Administrativa, 188, 139-182.

Pendás García, B. (1988). Gobierno y forma de gobierno de las comunidades autónomas. Reflexiones sobre el dogma de la homogeneidad y sus límites. Documentación Administrativa, 215, 85-136.

Perini, M. (2009). Le regole del potere: primato del Parlamento o del Governo? Turín: Giappichelli.

Pisaneschi, A. (2015). Brevi considerazioni su efficienza del Governo e riforme costituzionali. Rivista Associazione Italiana Costituzionalisti, 4.

Requejo Pagés, J. L. (2004). Las relaciones entre el Gobierno y las Cortes Generales. Revista Española de Derecho Constitucional, 73-89.

Revenga Sánchez, M. (1988). La formación del Gobierno en la Constitución española de 1978. Madrid: CEC. 
Ripollés Serrano, R. (2012). Artículo 170, 171 y 172. En R. Ripollés Serrano. Comentarios al Reglamento del Congreso de los Diputados (pp. 1165-1189). Madrid: Congreso de los Diputados.

Sánchez Agesta, L. (1991). Sistema politico de la Constitución española de 1978, 6. a ed. Madrid: Edersa.

Sánchez Navarro, A. J. (1995). Las minorias en la estructura parlamentaria. Madrid: CEC.

- (1997). La oposición parlamentaria. Madrid: Congreso de los Diputados.

Torres Muro, I. (1995). El refrendo de la propuesta real de candidato a la presidencia del Gobierno. Revista de Estudios Politicos, 88, 145-163.

(2016). El rey y la investidura del presidente del Gobierno. Agenda pública. (21-12016). Disponible en: http://agendapublica.es/el-rey-y-la-investidura-del-presidente-del-gobierno/.

Zbíral, R. (2011). The Rules and Practice of the Investiture Vote in the Czech Republic. International and Comparative Law Review, 11 (2), 43-62. 